\title{
Ultrasound Elicits Behavioral Responses through Mechanical Effects on Neurons and Ion Channels in a Simple Nervous System
}

\author{
ㄱan Kubanek, ${ }^{1}$ Poojan Shukla, ${ }^{1}$ Alakananda Das, ${ }^{1}$ @Stephen A. Baccus, ${ }^{2}$ and ${ }^{\circledR}$ Miriam B. Goodman ${ }^{1}$ \\ 'Department of Molecular and Cellular Physiology and ${ }^{2}$ Department of Neurobiology, Stanford, California 94305
}

Focused ultrasound has been shown to stimulate excitable cells, but the biophysical mechanisms behind this phenomenon remain poorly understood. To provide additional insight, we devised a behavioral-genetic assay applied to the well-characterized nervous system of Caenorhabditis elegans nematodes. We found that pulsed ultrasound elicits robust reversal behavior in wild-type animals in a pressure-, duration-, and pulse protocol-dependent manner. Responses were preserved in mutants unable to sense thermal fluctuations and absent in mutants lacking neurons required for mechanosensation. Additionally, we found that the worm's response to ultrasound pulses rests on the expression of MEC-4, a DEG/ENaC/ASIC ion channel required for touch sensation. Consistent with prior studies of MEC-4dependent currents in vivo, the worm's response was optimal for pulses repeated 300-1000 times per second. Based on these findings, we conclude that mechanical, rather than thermal, stimulation accounts for behavioral responses. Further, we propose that acoustic radiation force governs the response to ultrasound in a manner that depends on the touch receptor neurons and MEC-4-dependent ion channels. Our findings illuminate a complete pathway of ultrasound action, from the forces generated by propagating ultrasound to an activation of a specific ion channel. The findings further highlight the importance of optimizing ultrasound pulsing protocols when stimulating neurons via ion channels with mechanosensitive properties.

Key words: Caenorhabditis elegans; mechanosensation; mechanosensitive ion channels; neurostimulation; thermosensation; ultrasound

Significance Statement

How ultrasound influences neurons and other excitable cells has remained a mystery for decades. Although it is widely understood that ultrasound can heat tissues and induce mechanical strain, whether or not neuronal activation depends on heat, mechanical force, or both physical factors is not known. We harnessed Caenorhabditis elegans nematodes and their extraordinary sensitivity to thermal and mechanical stimuli to address this question. Whereas thermosensory mutants respond to ultrasound similar to wild-type animals, mechanosensory mutants were insensitive to ultrasound stimulation. Additionally, stimulus parameters that accentuate mechanical effects were more effective than those producing more heat. These findings highlight a mechanical nature of the effect of ultrasound on neurons and suggest specific ways to optimize stimulation protocols in specific tissues.

\section{Introduction}

Low-intensity, focused ultrasound affects the function of excitable cells in the central (Fry et al., 1958; Meyers et al., 1959; Foster and

Received June 1, 2017; revised Jan. 11, 2018; accepted Jan. 27, 2018.

Author contributions: J.K., A.D., S.A.B., and M.B.G. designed research;J.K., P.S., and A.D. performed research; J.K. analyzed data; J.K., A.D., S.A.B., and M.B.G. wrote the paper.

This work was supported by National Institutes of Health Grant K99NS100986 to J.K., Grants R01NS047715 and R01NS092099 to M.B.G., Stanford Neuroscience Institute and Mathers Foundation to M.B.G. and S.A.B., and a Stanford Medicine Dean's Fellowship to J.K. We thank our colleagues at Stanford, Zhiwen Liao, Michael Menz, Patrick Ye, and Martin Prieto for laboratory and technical assistance and input on the experimental design; and Merritt Maduke, Pierre Khuri-Yakub, Kimberly Butts-Pauly, and Julian Brown for helpful comments; Zachary Pincus (Washington University, St. Louis) for providing animals for initial pilot experiments; and Alessandro Sanzeni and Massimo Vergassola for additional model calculations.

The authors declare no competing financial interests.

Correspondence should be addressed to Dr. Miriam B. Goodman, B-111 Beckman Center, 279 Campus Drive, Stanford, CA 94305. E-mail: mbgoodman@stanford.edu.
Wiederhold, 1978; Gavrilov et al., 1996; Tufail et al., 2011; Yoo et al., 2011; Deffieux et al., 2013; King et al., 2013; Menz et al., 2013) and peripheral (Mihran et al., 1990; Tsui et al., 2005; Colucci et al., 2009) nervous systems, and the heart (Harvey, 1929; Buiochi et al., 2012). Because it propagates deep into tissue while retaining spatial focus, it has garnered considerable attention for its potential as a noninvasive tool for stimulation of the brain and the heart (Tufail et al., 2011; Lee et al., 2016). Despite these investigations, how ultrasound stimulates excitable cells has been a mystery since the discovery of its stimulatory effects $>8$ decades ago (Harvey, 1929).

Physical mechanisms for ultrasound-dependent tissue excitation have been divided into thermal and nonthermal effects ( $\mathrm{Da}$ - 
lecki, 2004; Naor et al., 2016; Sassaroli and Vykhodtseva, 2016; Ye et al., 2016). The latter category encompasses mechanical effects, such as membrane oscillation, cavitation, or radiation force. Ultrasound stimulation generally occurs under conditions that heat tissues by $<1^{\circ} \mathrm{C}$ (Tufail et al., 2010; Yoo et al., 2011; Menz et al., 2013). Nonetheless, even small temperature changes could activate certain classes of ion channels, such as temperaturesensitive TRP cation channels (Diaz-Franulic et al., 2016) and TREK potassium channels (Schneider et al., 2014). Moreover, the rate of temperature change may also contribute to the stimulatory effects (Rabbitt et al., 2016).

The mechanical effects of ultrasound could be converted into ionic currents and changes in electrical excitability by increasing mechanical strain in a manner that directly activates ion channels (Tyler, 2011) or by changes in membrane thickness and capacitance (Plaksin et al., 2014). For instance, increases in membrane tension are thought to catalyze conformational change and activate ion channels in which the open state has a larger cross-sectional area than the closed state (Sukharev and Corey, 2004). Thus, if ultrasound stimulation were to change membrane tension, then it could alter the activity of mechanosensitive channels, including voltage-gated sodium and potassium channels that exhibit membrane tension-dependent gating (Beyder et al., 2010; Brohawn et al., 2014). Consistent with this idea, ultrasound stimulation has been shown to increase currents carried by two-pore domain (K2P) potassium channels and voltage-gated sodium channels expressed in Xenopus oocytes (Kubanek et al., 2016).

This body of work has raised the question whether or not ultrasound affects nervous systems via thermal or nonthermal effects (Iversen et al., 2017) and, if nonthermal effects are dominant, how is ultrasound transduced into membrane strain (tension) and channel activation. To contribute additional insight into these questions, we developed a behavioral-genetic assay using Caenorhabditis elegans roundworms. The nervous system of this animal has been characterized in its entirety, the animal performs simple behaviors that are easily monitored using video tracking and machine vision tools (Husson et al., 2005), and the model is amenable to experimental studies involving tens to hundreds of individual animals. Two additional aspects of the worm's biology are exploited in this study. First, C. elegans has extraordinarily sensitive thermosensory and mechanosensory neurons able to detect thermal fluctuations of $\leq 0.05^{\circ} \mathrm{C}$ (Ramot et al., 2008) and applied forces of $50 \mathrm{nN}$ (O’Hagan et al., 2005), respectively. Second, mutants exist in which thermosensory and mechanosensory neurons are disabled independently.

We found that pulsed ultrasound stimulation evokes avoidance responses whose probability increases with acoustic pressure and stimulus duration and shows optima for specific ultrasound pulsing protocols. Mutants lacking neurons required for thermosensation responded similar to wild-type animals, whereas those lacking neurons or ion channels required for mechosensation failed to mount avoidance responses to ultrasound stimulation. The mechanical nature of the effect led us to a detailed characterization of the involved neurons and ion channels as well as a characterization of the optimal stimulation parameters.

\section{Materials and Methods}

Animals and strains. The following strains were used in this study: N2 (RRID:WB-STRAIN:N2(ancestral));CB1338 mec-3(e1338) IV (RRID: WB-STRAIN:CB1338); CB1611 mec-4(e1611) X (RRID:WB-STRAIN: CB1611); TU253 mec-4(u253) X (RRID:WB-STRAIN:TU253); IK597 $g c y-23(n j 37) g c y-8($ oy44)gcy-18(nj38) IV (RRID:WB-STRAIN:IK597);
VC1141 trp-4(ok1605) I (RRID:WB-STRAIN:VC1141);VC818 trp-4(gk341) I (RRID:WB-STRAIN:VC818); TQ296 trp-4(sy695) I (RRID:WBSTRAIN:TQ296); GN716 trp-4(ok1605) I, outcrossed four times from VC1141. All mutants were derived from the N2 (Bristol) background, which serves as the wild-type strain in this study. Except for GN716, which was prepared explicitly for this study, and TQ296, which was a gift of X.Z.S. Xu (University of Michigan), strains were obtained either from a frozen repository maintained in our laboratory or from the Caenorhabditis Genetics Center.

The three trp-4 alleles we studied all encode deletion or null alleles of the $\operatorname{trp}-4$ gene, which encodes the key pore-forming subunit of a mechanosensory ion channel expressed in the CEP texture-sensing neurons (Kang et al., 2010). TRP-4 is orthologous to the mechanically-gated NOMPC channel from Drosophila.

The ok1605 allele encodes an in-frame, $1 \mathrm{~kb}$ deletion that removes exons $12-14$ of the trp-4 gene and is predicted to result in the loss of ankyrin repeats 16-21 from the TRP-4 protein. The $g k 341$ allele contains a small deletion encompassing exon 2 and is predicted to cause a frameshift in the transcript and the introduction of an early stop codon. The sy695 allele contains an unmapped $3 \mathrm{~kb}$ deletion in the $3^{\prime}$ region of the gene. This deletion is thought to disrupt the transmembrane poreforming domain of TRP-4 (Li et al., 2006). The GN716 trp-4(ok1605) strain was derived by outcrossing VC1141 trp-4(ok1605) with wild-type (N2) in four rounds. We used PCR to verify that all $\operatorname{trp}-4$ mutant strains harbored the expected genetic deletions in the trp- 4 locus.

Imaging and transducer control. For each assay, we transferred a single adult animal from a growth plate to a 4-mm-thick NGM agar slab that was free of bacteria. Because the agar slab consists of a $2 \%$ agar solution in saline, it is a good approximation of the acoustic properties of many biological tissues (Altman et al., 1974). To retain animals within the camera's field of view, we created a boundary consisting of a filter paper ring saturated by a copper sulfate $(500 \mathrm{~mm})$ solution, as described previously (Ramot et al., 2008).

To deliver ultrasound stimuli, we used a commercially available piezoelectric ultrasonic transducer (A327S-SU-CF1.00IN-PTF, Olympus, 1-inch line-focused) positioned 1 inch $(2.54 \mathrm{~cm})$ below the top of the agar slab and oriented perpendicular to the surface of the agar slab (see Fig. 1A). We filled the space between the transducer surface and the bottom of the agar slab with degassed water, contained within a plastic cone mounted on the transducer. The water was degassed by boiling for $30 \mathrm{~min}$ and stored in air-tight tubes. The slab did not appear to attenuate ultrasound pressure, according to measurements with a hydrophone (data not shown).

We used oblique illumination via a circular array $(20 \mathrm{~cm}$ in diameter $)$ of red LEDs to provide the optical contrast between animals and the surface of the agar slab needed to track animal movement using the Parallel Worm Tracker (Ramot et al., 2008). The image was magnified $3 \times$ (zoom lens, Navitar). The camera's chip FOV was $5.6 \times 4.2 \mathrm{~mm}$. Image contrast was optimal when the plane of the LED array was $\sim 1 \mathrm{~cm}$ above the top of the agar slab. We also used a blue LED, controlled by an Arduino Uno board and mounted $5 \mathrm{~cm}$ above the agar slab, to deliver an optical signal synchronized to the stimulus onset.

To generate signals driving the ultrasound transducer, we used a function generator (HP 8116A, Hewlett-Packard) controlled by an Arduino Uno board and an amplifier with a gain of $50 \mathrm{~dB}$ (ENI-240L, ENI). The acoustic pressures we generated were measured in free field using a calibrated hydrophone (HGL-0200, Onda) combined with a preamplifier (AG-2020, Onda). The hydrophone measurements were performed at the peak spatial pressure. The hydrophone manufacturer's calibration values around the frequency of $10 \mathrm{MHz}$ were steady and showed only minimal level of noise.

Behavioral recordings. For each trial, a freely moving animal was monitored via a digital video camera (SME-B050-U, Mightex) operated in a live-video mode until it approached the ultrasound focus head first. We started recording videos $\sim 5 \mathrm{~s}$ before the predicted approach to the ultrasound focus and continued recording for $\sim 10$ s following the delivery of each stimulus. Each individual animal was tested in 10 trials with an intertrial interval of at least $20 \mathrm{~s}$. All animals were hermaphrodites, assayed blind to genotype and as adults. We applied stimuli at the follow- 
ing pressures (in $\mathrm{MPa}$ ) by controlling the output of the function generator (voltage in $\mathrm{mV}$, shown in parentheses 0 (also known as sham treatment, the amplifier was operated but not connected to the transducer), 0.2 (60), 0.4 (120), 0.6 (180), 0.8 (240), and 1.0 (300). We were not able to deliver stimuli more intense than $1.0 \mathrm{MPa}$ because the transducer was damaged by sustained operation at this level. The protocol for determining the effects of stimulus duration, duty cycle, and pulse repetition frequency (PRF) was analogous, except that we varied the levels of the respective quantities.

Each animal's movement was recorded at 20 frames per second at a resolution of $576 \times 592$ pixels. We recorded $\sim 350$ frames per video. The resolution and frame rate were chosen to be high enough to provide reliable movement characterization while maintaining acceptable size of the stored videos.

Quantification of response frequency and baseline response frequency. To detect bona fide ultrasound-evoked reversals, we measured the velocity vector over the interval from $250 \mathrm{~ms}$ to $1 \mathrm{~s}$ following the ultrasound onset and that during a $1 \mathrm{~s}$ period immediately preceding the ultrasound onset. Next, we computed the vector difference and evaluated the magnitude of that difference. We asked whether this metric differed from the null distribution constructed over all baseline recordings (same time windows, just shifted $1 \mathrm{~s}$ back in time so that there could be no effect of ultrasound) available for a given animal. If the vector difference was unlikely $(p<0.01)$ to have been drawn from the null distribution, we classified the response as a reversal. We computed the proportion of significant responses over the 10 stimulus repetitions for each animal and refer to this metric as the response frequency.

The computation of the baseline response frequency (dashed lines in the plots) was analogous to the computation of the response frequency with the exception that the metrics were taken in time windows before the ultrasound could have any impact (i.e., before the ultrasound was turned on). In particular, the velocity difference was computed by comparing a $1 \mathrm{~s}$ time window immediately preceding the ultrasound to a $1 \mathrm{~s}$ time window preceding the ultrasound onset by $1 \mathrm{~s}$. The baseline distribution used the same time windows, just shifted back in time by $1 \mathrm{~s}$. The baseline response frequency was indistinguishable across the tested animal strains $\left(F_{(4,95)}=0.28, p=0.90\right.$, one-way ANOVA).

Simulation of the relationship between reversal frequency and duty cycle. The simulation shown in Figure $5 B$ was derived as follows. First, we assumed that the signal relevant to modulation of reversal behavior is the envelope modulating the carrier frequency of the ultrasound transducer. Next, for each duty cycle value we tested, we converted this signal into the frequency domain using the function $\mathrm{ftt}$ in MATLAB (The MathWorks). Finally, this signal was convolved with the filter computed by Eastwood et al. (2015), and the effective (root mean squared or rms) value of the resulting signal was taken as the model's output. Thus, this simulation rests on the delivered stimulus waveform and the previously proposed mechanical filter. The only adjustment was a linear scaling factor used to plot the results on common graph. We note that the filter reported by Eastwood et al. (2015) was defined over the range from $1 \mathrm{~Hz}$ to $3 \mathrm{kHz}$, which we extrapolated to $10 \mathrm{kHz}$.

Temperature measurements. We used a dual sensing fiberoptic hydrophone (Precision Acoustics) to measure temperature (Morris et al., 2009). This device uses optical interferometry to record acoustically and thermally induced changes in thickness of its sensing membrane by ultrasound. When performing the measurements, we immersed the tip of the optic fiber into the agar at the location of maximal pressure. The device, which recorded the temperature at a $200 \mathrm{~Hz}$ sampling rate, rapidly registered the changes in temperature to ultrasound onset. As expected, temperatures were highest at the end of the ultrasound burst; consequently, we report the difference in temperature between the start and end of the ultrasound burst.

Simulation of acoustic radiation force. We used the k-Wave simulation tool (Treeby and Cox, 2010) to estimate the acoustic radiation force that acted on the animals in our setup. The simulation used the same geometry and media as our setup, including water, agar (4-mm-thick), worm on the agar $(0.05 \times 0.05 \times 1.0 \mathrm{~mm})$, and air. The speed of sound $\left(\mathrm{m} \mathrm{s}^{-1}\right)$, density $\left(\mathrm{kg} \mathrm{m}^{-3}\right)$, and acoustic attenuation coefficient $\left(\mathrm{dB} \mathrm{cm} \mathrm{cm}^{-1}\right.$ $\mathrm{MHz}^{-1}$ ) for these media were, respectively, set to the following: (1540,
$1000,0.0022),(1548,1000,0.40),(1562,1081,1.2)$, and $(343,1.2,1.6)$ (Barber et al., 1970; Kremkau et al., 1981). The simulation grid was computed in steps of $1 \mathrm{~ns}$ in time and $10 \mu \mathrm{m}$ in space. We set the stimulus level such that the simulated pressure field had an amplitude of $1 \mathrm{MPa}$ at focus. We obtained the steady-state time-average radiation force density field from the steady-state time-average intensity field provided by the simulation. To obtain the net radiation force, we integrated the force density field over a volume that approximated the animal's head $(0.05 \times$ $0.05 \times 0.2 \mathrm{~mm})$. This resulted in a net force magnitude of $873 \mathrm{nN}$. Integrating the force density over a larger volume had only a small influence on the results because the force field was strongest near the animal's head (see Fig. $1 A$ ). In Figure $1 B$, the force density is integrated within cubes of $0.05 \times 0.05 \times 0.05 \mathrm{~mm}$, using acoustic parameters of the worm.

Experimental design and statistical analysis. The C. elegans hermaphrodites used in this study were age-synchronized by hypochlorite treatment (Stiernagle, 2006) and cultivated at $15^{\circ} \mathrm{C}$ or $20^{\circ} \mathrm{C}$. There were no detectable effects of cultivation temperature, ambient temperature, or humidity on the ultrasound-evoked responses, which were collected over a period of months by two members of the research team. All behavioral recordings were performed blind to genotype, and we determined whether or not a given trial included a reversal event as described above. The response rate was computed as the fraction of 10 trials that evoked a reversal, and results were pooled from $n=20$ C. elegans subjects.

We used ANOVAs to assess the effect of stimulus pressure, duration, PRF, and duty cycle. In this linear model (see Figs. 3, 5), the dependent variable is the response frequency as described above. The independent variable (the one factor) is pressure (see Fig. 3), or PRF or duty cycle (see Fig. 5). We report the $p$ values as well as the $F$ statistic and the degrees of freedom of the tests.

\section{Results}

As a first step toward determining how animals and excitable tissues detect and respond to ultrasound stimulation, we placed single adult wild-type (N2) nematodes on sterile agar slabs and tracked their movement using a digital video camera and the Parallel Worm Tracker (Ramot et al., 2008), adapted for tracking single animals (Fig. 1A). We subjected each animal to pulsed ultrasound (10 MHz frequency, $200 \mathrm{~ms}$ duration, $1 \mathrm{kHz}$ pulse repetition frequency (PRF) at 50\% duty cycle; Fig. $1 B$ ) when it approached the ultrasound focus and found that this stimulus elicits similar reversal behaviors over the course of 10 trials (Fig. 2 ). The effect was due to ultrasound stimulation per se because sham stimuli $(0 \mathrm{MPa})$ did not increase reversals above their basal or unstimulated rate (Fig. $2 A, B$ ).

Behavioral responses were robust across trials for a given individual and among all animals tested (Fig. 2B). We determined whether each animal's response to ultrasound stimulation was statistically different from spontaneous changes in direction (see Materials and Methods). For each animal, we quantified the proportion of significant responses over the 10 stimulus trials, and refer to this metric as response frequency.

The response frequency increased with ultrasound pressure (Fig. 2C) and was indistinguishable from the spontaneous reversal rate for the sham stimulus (Fig. $2 A$, dotted line; $p=0.52, t$ test, $n=20)$. The reversal rate increased above the baseline at a pressure of $0.6 \mathrm{MPa}\left(p<10^{-6}\right)$. At $1 \mathrm{MPa}$ (Fig. $2 B$ ), there was a significant response in $77.5 \%$ of trials, on average. The relationship between response frequency and applied pressure was welldescribed by a sigmoid function as follows:

$$
F=\frac{F_{\max }}{1+\exp \left(\frac{P-P_{1 / 2}}{\text { slope }}\right)}+\text { base }
$$

where $F$ is the response frequency, $P$ is the pressure, and base is the spontaneous reversal frequency. 
For wild-type animals stimulated by a $200 \mathrm{~ms}$ pulse (1 kHz PRF, 50\% duty cycle), the best fit parameters were $F_{\max }=$ $83 \% ; P_{1 / 2}=0.71 \mathrm{MPa}$, slope $=0.15$, base $=5 \%$. Thus, the half-activation pressure equals $0.71 \mathrm{MPa}$. A one-way ANOVA also detected a significant modulation of the response frequency by pressure $\left(F_{(5,114)}=\right.$ 103.4, $\left.p<10^{-39}\right)$, reinforcing the idea that the probability of ultrasound-induced reversal depends on stimulus pressure.

We also tested the effect of varying the total duration of the ultrasound stimulus (Fig. 2D), holding all other parameters (i.e., pressure, duty cycle, PRF) constant. In agreement with a previous study (Ibsen et al., 2015), responses were weak or absent when the stimulus was brief. There was a significant modulation of the response frequency by stimulus duration (one-way ANOVA, $F_{(3,76)}=30.8, p<$ $\left.10^{-12}\right)$. Stimuli of $100 \mathrm{~ms}$ in duration or longer produced substantial effects. The response frequency did not increase substantially beyond stimulus duration of $200 \mathrm{~ms}$ (response frequency at $200 \mathrm{~ms}$ vs 400 ms: $p=0.24$, paired $t$ test, $n=20$ ). Therefore, we used a stimulus duration of $200 \mathrm{~ms}$ for subsequent experiments.

In principle, ultrasound-evoked behaviors could depend on thermosensation, mechanosensation, or both. We used a genetic approach to distinguish among these possibilities, leveraging mutants deficient in thermosensation or mechanosensation. To test for thermal effects, we compared ultrasound-evoked behaviors in wild-type and $g c y$-23(nj37)gcy-8(oy44)gcy-18(nj38) that lack a trio of receptor guanylate cyclases expressed exclusively in the AFD thermoreceptor neurons and are defective in thermotaxis (Garrity et al., 2010; Glauser and Goodman, 2016). Although these mutants have an intact AFD thermoreceptor neuron, they lack the ability to sense tiny $\left(<0.05^{\circ} \mathrm{C}\right)$ thermal fluctuations in temperature (Ramot et al., 2008; Wasserman et al., 2011). As shown in Figure $3 A$, the response of these thermosensory-defective mutants was indistinguishable from that of wild-type animals. The mutants retained modulation by stimulus pressure, as assessed by one-way ANOVA $\left(F_{(5,114)}=80.7, p<10^{-35}\right)$. Furthermore, as expected from the plot, a two-way ANOVA with factors animal strain and pressure failed to detect a significant difference between the strains $\left(F_{(1,228)}=0.02, p=0.89\right)$ as well as the strain $\times$ pressure interaction $\left(F_{(5,228)}=1.40, p=0.23\right)$. Thus, the ability to sense tiny $\left(<0.05^{\circ} \mathrm{C}\right)$ thermal fluctuations is not required for ultrasoundinduced reversal behaviors.

Having established that thermosensation is dispensable for ultrasound-evoked reversals, we compared responses in wildtype animals and mutants defective in mechanosensation. Specifically, we sought to quantify ultrasound-evoked responses in mutants in which selected mechanoreceptor neurons fail to properly differentiate during development, degenerate, or lack essential, pore-forming subunits of known sensory mechano-
B

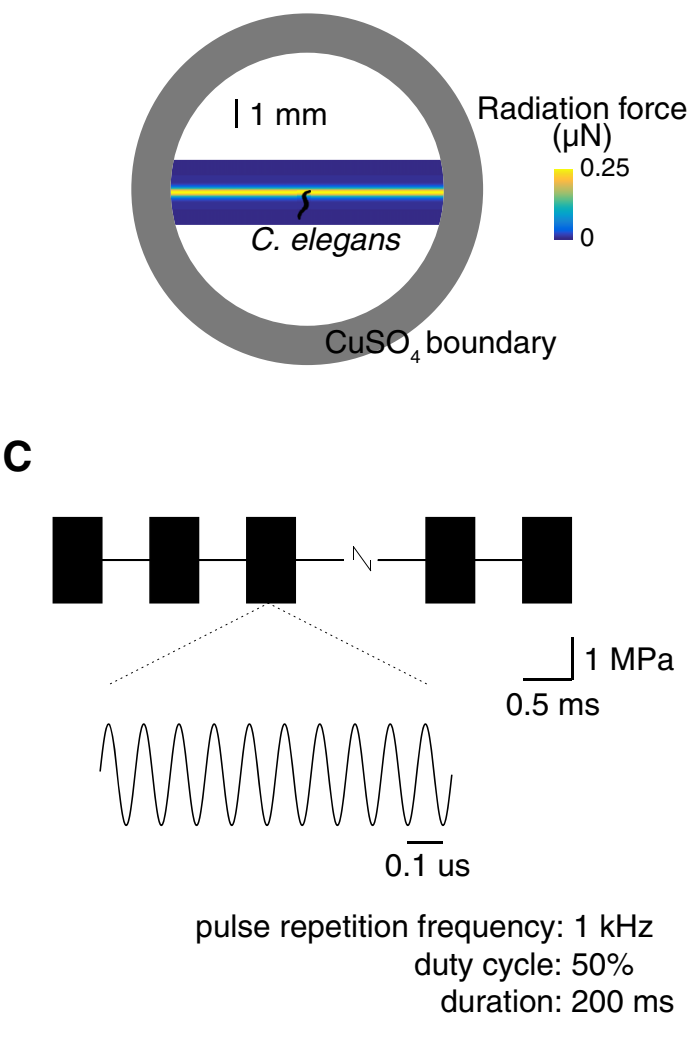

Figure 1. A system for delivering pulsed ultrasound to C. elegans nematodes. $\boldsymbol{A}$, Schematic side view of the setup, showing a single wild-type adult hermaphrodite crawling on the surface of agar slab, tracked by a digital video camera, and maintained within the field of view by a copper sulfate boundary. A piezoelectric ultrasound transducer (10 $\mathrm{MHz}$ carrier frequency, lineradiation force expected from the line-focused $10 \mathrm{MHz}$ ultrasound transducer (see Materials and Methods). Animals were stimu-

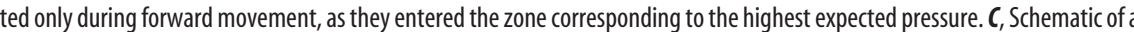
typical stimulus consisting of ultrasound pulses delivered at PRF of $1 \mathrm{kHz}$ for a total duration of $200 \mathrm{~ms}$ at $50 \%$ duty cycle. In this study, we systematically varied the applied pressure, pulse duration, PRF, and duty cycle.

electrical transduction channels: MEC-4 (O'Hagan et al., 2005), TRP-4 (Kang et al., 2010). The goal of these experiments was to identify the neurons most likely to serve as the first responders to ultrasound stimulation and to determine whether or not such sensitivity relied upon known mechano-electrical transduction channels.

The mec-3(e1338) mutants fail to generate three sets of neurons known to participate in gentle and harsh touch sensation and are insensitive to both gentle and harsh touch (Way and Chalfie, 1989). The six touch receptor neurons (TRNs) are required for sensing gentle touch, and the two pairs of multidendritic PVD and FLP neurons act as polymodal sensors of mechanical and nociceptive stimuli (Schafer, 2015). We found that mec-3 mutants are insensitive to ultrasound stimulation (Fig. $3 B$ ): the mec-3 mutants showed no significant modulation of the response frequency by pressure $\left(F_{(5,114)}=1.18, p=0.32\right.$, one-way ANOVA). A two-way ANOVA detected both a highly significant difference between the strains $\left(F_{(1,228)}=246.1, p<\right.$ $\left.10^{-37}\right)$ and a highly significant strain $\times$ pressure interaction $\left(F_{(5,228)}=56.8, p<10^{-37}\right)$. This effect was specific to ultrasound-evoked reversals; mutants moved at an average speed 

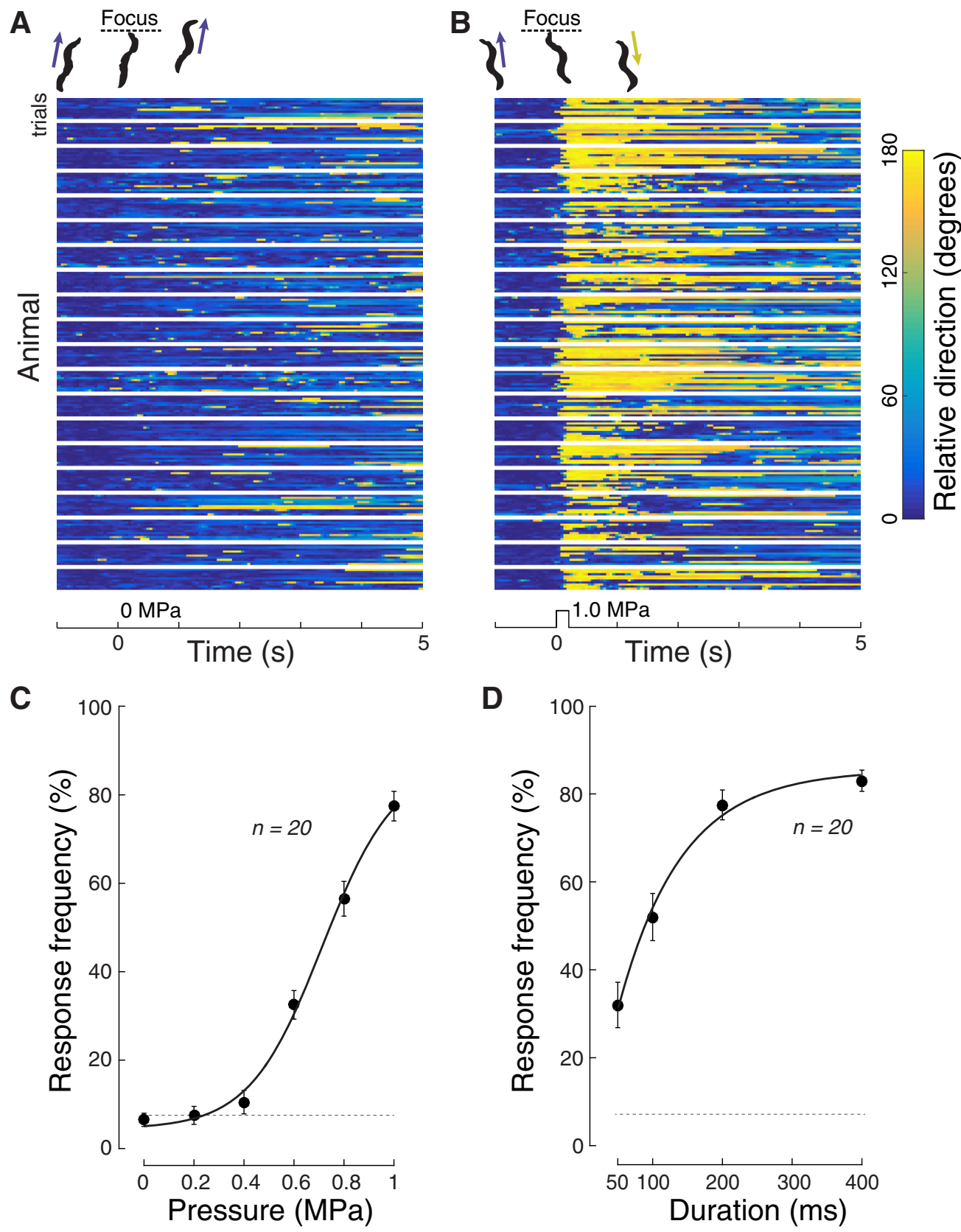

Figure2. Ultrasound elicits reversal behavior in a pressure-and stimulus time-dependent manner in wild-type C. elegans. $A, B$, Raster plots showing the response of 20 animals (10 trials/animals) to a $200 \mathrm{~ms}$ sham stimulus (0 MPa pressure, $\boldsymbol{A}$ ) and a bon a fide stimulus $(1.0 \mathrm{MPa}, \boldsymbol{B})$. Heading angle is encoded in color such that headings similar to the average angle in the $1 \mathrm{~s}$ window immediately preceding stimulus onset are blue and reversals are encoded in yellow. Rows correspond to single trials and blocks are 10 trials delivered to each animal; traces were smoothed with a zero-lag rectangular sliding $150 \mathrm{~ms}$ window. Top, Silhouettes depict representative responses to sham $(\boldsymbol{A})$ and $1.0 \mathrm{MPa}$ stimuli $(\boldsymbol{B})$. $\boldsymbol{C}$, Reversal frequency increases with applied pressure. Points indicate mean $\pm \operatorname{SEM}(n=20)$ for animals stimulated at each of the six pressure values for a total of 10 trials. Solid line indicates a Boltzmann fit to the data with an $P_{1 / 2}$ of $0.71 \mathrm{MPa}$, a slope factor of 0.15 , and a maximum probability of $83 \%$. Dotted line indicates the unstimulated reversal rate. Stimulus parameters: $1 \mathrm{kHz}, 50 \%$ duty cycle, $200 \mathrm{~ms}$ pulse duration, variable pressure. $\boldsymbol{D}$, Reversal probability increases with stimulus duration. Points indicate mean \pm SEM $(n=20)$. Smooth line indicates an exponential fit to the data with a time constant of 90 ms. Stimulus parameters: $1 \mathrm{kHz}, 50 \%$ duty cycle, variable pulse duration, 1.0 MPa pressure. $\boldsymbol{C}, \boldsymbol{D}$, Dotted line indicates baseline rate of responding (see Materials and Methods). Smooth line indicates an exponential fit to the data with a time constant of $90 \mathrm{~ms}$.

that was indistinguishable from wild-type animals (speed measured during $1 \mathrm{~s}$ period preceding ultrasound onset; wild-type: $0.21 \mathrm{~mm} / \mathrm{s} ;$ mec-3: $0.17 \mathrm{~mm} / \mathrm{s} ; p=0.11, n=20, t$ test). These average speeds are within the range of values reported previously for wild-type animals (Ramot et al., 2008). This result shows that the mec-3-dependent mechanoreceptor neurons are required for ultrasound-evoked reversals and suggests that ultrasound can exert forces on neural tissue sufficient to activate these neurons.
We narrowed the search to a subset of the mec-3-dependent mechanoreceptor neurons by testing ultrasound-evoked behavior in mec-4(e1611) mutants in which the six TRNs degenerate and the PVD and FLP mechanoreceptor neurons are intact (Driscoll and Chalfie, 1991). As found in mec-3 mutants, ultrasound failed to evoked reversals in mec-4(e1611) (Fig. 3C), and there was no significant modulation of the response frequency by the ultrasound pressure amplitude in these animals 
A

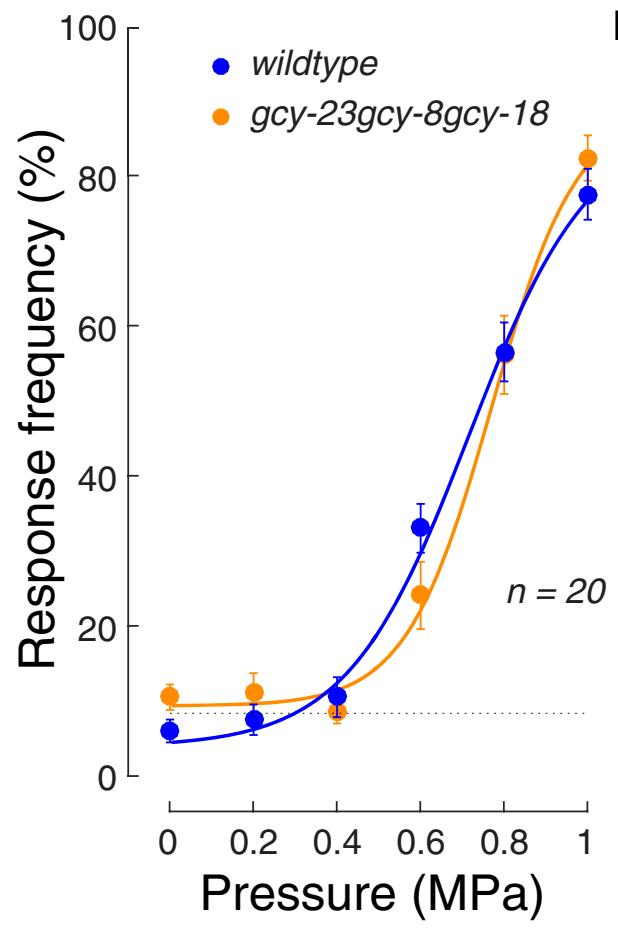

C

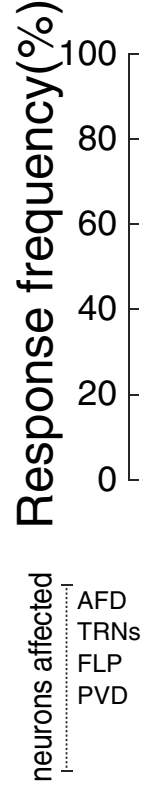

B

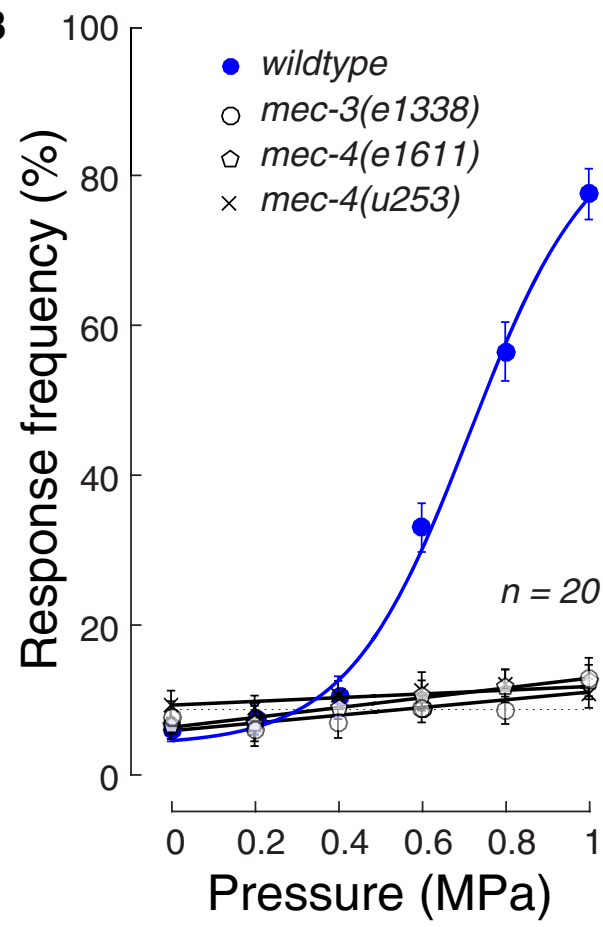

pulse frequency: $1 \mathrm{kHz}$

duty cycle: $50 \%$

duration: $200 \mathrm{~ms}$

pressure: $1 \mathrm{MPa}$

$n=20$

Figure 3. Loss of mechanosensation, but not thermosensation, disrupts ultrasound-evoked reversals. $\boldsymbol{A}, \boldsymbol{B}$, Pressure-response curves of wild-type N2 animals (blue) compared with a thermosensation-defective mutant (orange) and three mechanosensation-defective mutants (black): mec-3(e1338), mec-4(e1611), and mec-4(u253). Points indicate mean \pm SEM ( $n=$ 20 animals tested in 10 trials/animals). Smooth lines indicate fit to the data according to a sigmoidal function. The data and fit for wild-type are the same as in Figure $2 C$. Fitting parameters for $g c y-23(n j 37) g c y-8($ oy 44$) g c y-18(n j 38)$ are $\left(F_{\max ,} P_{1 / 2}\right.$ slope, base): $80 \%, 0.76 \mathrm{MPa}, 0.10,9 \%$. Dotted lines indicate the average baseline response rate (see Materials and Methods) for each case. There was no significant effect of genotype on baseline reversal rates. Stimulus parameters: $1 \mathrm{kHz}$ pulse frequency, $50 \%$ duty cycle, $200 \mathrm{~ms}$ duration, variable pressure. $\boldsymbol{C}$, Response rate as a function of genotype. Error bars indicate mean $(S E M ; n=20)$ reversal rate. Annotations below the graph indicate the nature of the known sensory deficit associated with each genotype. Animals were tested as young adult hermaphrodites and blind to genotype.

(Fig. $\left.3 C ; F_{(5,114)}=1.47, p=0.20\right)$. Moreover, a two-way ANOVA detected a significant difference between the mutant and wildtype strains and a highly significant strain $\times$ pressure interaction (both $p<10^{-36}$ ). Thus, the TRN neurons, which can detect forces as small as $50 \mathrm{nN}$ (O'Hagan et al., 2005), are required for behavioral responses to ultrasound stimulation in C. elegans.

Next, we investigated proteins expressed in the TRN neurons that might mediate the effect. Of particular interest, the TRNs express mec-4, which encodes a non-voltage-gated sodium channel of the DEG/ENaC/ASIC family required for touch-evoked reversals. MEC-4 is expressed exclusively in the TRNs and is an essential pore-forming subunit of the mechanosensitive ion channel activated by mechanical loads applied directly to the animal's skin (O'Hagan et al., 2005). Like mec-3 and mec-4(e1611) mutants, mec-4(u253) null mutants are insensitive to ultrasound stimulation (Fig. 3D). These animals have intact TRNs but lack 

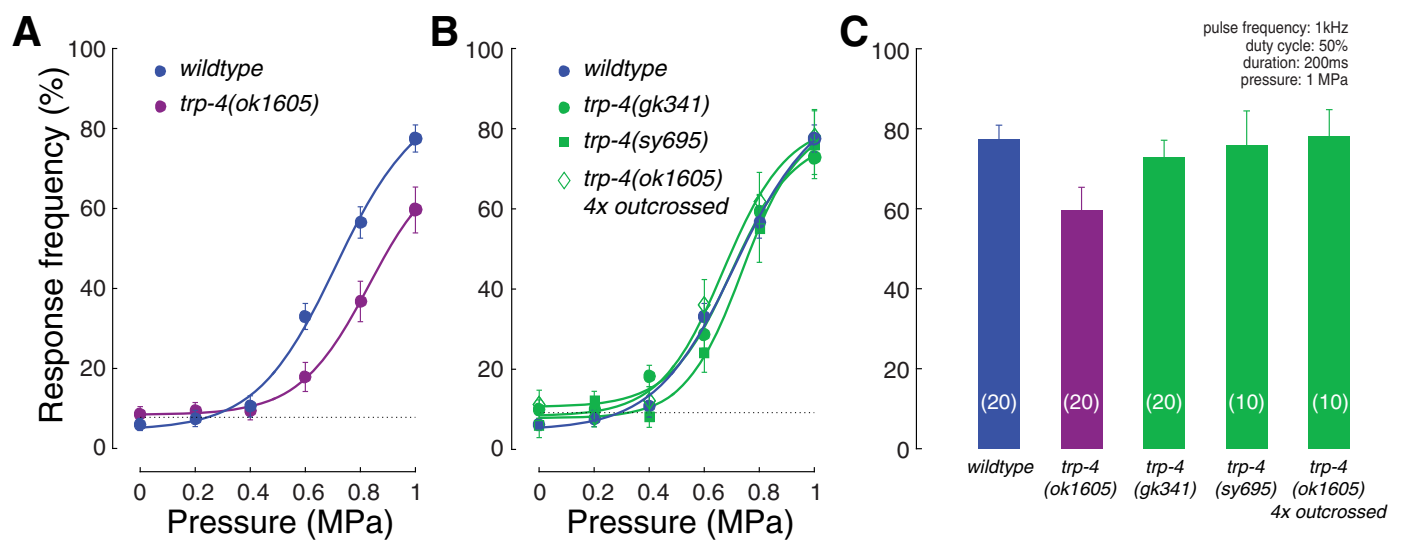

Figure 4. Strains carrying deletions in the trp-4 NOMPC channel gene differ in their response to ultrasound stimulation. $A$, Pressure-response curves of wild-type (blue), VC1141 trp-4(ok1605) (magenta) mutants used in a previous study (lbsen et al., 2015). Smooth curve fit to the trp-4(ok1605) data yielded $F_{\max }=65 \% ; P_{1 / 2}=0.83 \mathrm{MPa}$, slope $=0.13$, base $=8 \%$. $\boldsymbol{B}$, Pressure response curves of three other trp-4 deletion mutants were indistinguishable from wild-type. VC818 trp-4(gk341), TQ296 trp-4(sy695), and GN716 trp-4(ok1605) mutants, which was derived from VC1141 by outcrossing four times with wild-type (N2) animals. C, Response rate in four trp-4 mutant strains. Error bars indicate mean \pm SEM reversal rate evoked by ultrasound stimulation with the following parameters: $1 \mathrm{kHz}, 50 \%$ duty cycle, $200 \mathrm{~ms}$ pulse duration, $1.0 \mathrm{MPa}$. Dotted line indicates the average baseline response rate (see Materials and Methods). The number of animals analyzed across 10 trials is indicated in parentheses. We used PCR to verify that all strains harbored the expected deletions in the trp-4 locus (Materials and Methods). Wild-type data are from Figure $2 C$.

the MEC-4 protein required for mechanotransduction and showed no significant modulation of the response frequency by the ultrasound pressure $\left(F_{(5,114)}=0.37, p=0.87\right)$. A two-way ANOVA revealed a significant effect of genotype $X$ pressure interaction (both $p<10^{-35}$, two-way ANOVA). Although the response of Mec mutants appeared as if it might be modulated by pressure (Fig. $3 B-D$ ), this apparent modulation was not significant $(p>0.09$, one-way ANOVA). Collectively, these results establish that behavioral responses to focused ultrasound depend on the TRN neurons and the MEC-4 protein.

Thus far, we have shown that focused ultrasound evokes reversal behaviors in freely moving C. elegans nematodes in a pressure- and stimulus duration-dependent manner (Fig. 2) and that such responses depend on the animal's ability to detect mechanical, but not thermal, stimuli (Fig. 3). Together, these findings imply that ultrasound exerts its effects on excitable tissues via a nonthermal, mechanical mechanism. Although additional work is needed to determine how ultrasound produces these effects, a leading possibility is the generation of mechanical strain in neurons expressing mechanosensitive ion channels, such as MEC-4.

MEC-4 is not the only protein thought to form a mechanosensitive ion channel in C. elegans nematodes. The TRP-4 protein is expressed in the CEP mechanoreceptor neurons and is an ortholog of the Drosophila NOMPC channel (Li et al., 2006) known to form mechanosensitive ion channels (Yan et al., 2013). A previous study showed that $C$. elegans responds to ultrasound-induced cavitation of microbubbles and proposed that these responses were TRP-4-dependent (Ibsen et al., 2015). To determine whether TRP-4 also contributed to ultrasound-evoked behaviors elicited in the absence of microbubbles, we analyzed the same trp-4 strain used by Ibsen et al. (2015): VC1141 trp-4(ok1605). In agreement with the prior report (Ibsen et al., 2015), we observed a modest deficit in ultrasound-evoked behavior (Fig. 4A). A two-way ANOVA detected both a main effect of strain $\left(F_{(1,228)}=17.8, p<0.0001\right)$ and a significant strain $\times$ pressure interaction $\left(F_{(5,228)}=4.8, p=\right.$ $0.0003)$. The defect in these mutants was not specific for ultrasound-evoked behaviors, however: trp-4 mutants had a lower average speed than wild-type mutants under baseline conditions ( 0.17 vs $0.21 \mathrm{~mm} / \mathrm{s}, p=0.0086$, $t$ test, $n=20$ ).

Because the ok1605 allele encodes a partial in-frame deletion and because we also observed that these mutants grew slowly compared with wild-type animals, we tested two additional deletions in the trp-4 gene: $g k 341$ and sy695. All three alleles (ok1605, $g k 341$, and sy695) encode deletions in the trp-4 gene, which we verified by PCR analysis of genomic DNA (see Materials and Methods). Despite the expectation that that the three trp-4 alleles would have the same ultrasound phenotype, we found that $g k 341$ and sy695 mutants responded to ultrasound just like wild-type animals (Fig. 4B; two-way ANOVAs, main effects and interactions, $p>0.29$ ).

These findings suggested that the deficit in the VC1141 trp-4(ok1605) animals might be due to a mutation present in the genetic background. We tested this idea by outcrossing the trp-4(ok1605) animals against wild-type (N2) animals four times while tracking the $\operatorname{trp}-4$ mutation via PCR. Animals from this new strain, GN716 trp-4(ok1605), had ultrasound-evoked behaviors that were indistinguishable from wild-type (Fig. $4 B$; two-way ANOVA, main effect and interaction, $p>0.23$ ). These results are summarized for the pressure of $1 \mathrm{MPa}$ in Figure $4 C$ and suggest that the defect we and others (Ibsen et al., 2015) have observed in VC1141 trp-4(ok1605) animals is due to mutation/s in the genetic background of this strain.

The finding that mechanosensation is an essential component of the behavioral effects of ultrasound suggests that there might be an optimal frequency of the ultrasound delivery that matches the mechanical properties of the tissue. We investigated this possibility by varying PRFs in the range from $30 \mathrm{~Hz}$ to $10 \mathrm{kHz}$ while keeping pulse duration, pressure, and duty cycle constant. We found that ultrasound indeed evoked reversals in a PRF-dependent manner (Fig. 5A). Response increased with PRF, reached a maximal value in the range of $300-1000 \mathrm{~Hz}$, and decreased at higher frequencies. The shape of the curve is reminiscent of the prediction (Fig. $5 A$, green) of a model linking indentation to mechanical strain and MEC-4-dependent channel activation (Eastwood et al., 2015). We note that, because stimuli were delivered at 50\% duty cycle at all the tested frequencies, the same amount of energy was delivered at all PRFs. If the behavioral responses were the result of tissue heating, little or no modulation by the PRF would be expected. Yet, the plot shows, and an ANOVA confirms, a strong modulation of the response by the $\operatorname{PRF}\left(F_{(5,114)}=10.8, p<10^{-7}\right)$.

We further hypothesized that discrete pulses may be more potent in eliciting mechanical effects because discrete pulses de- 

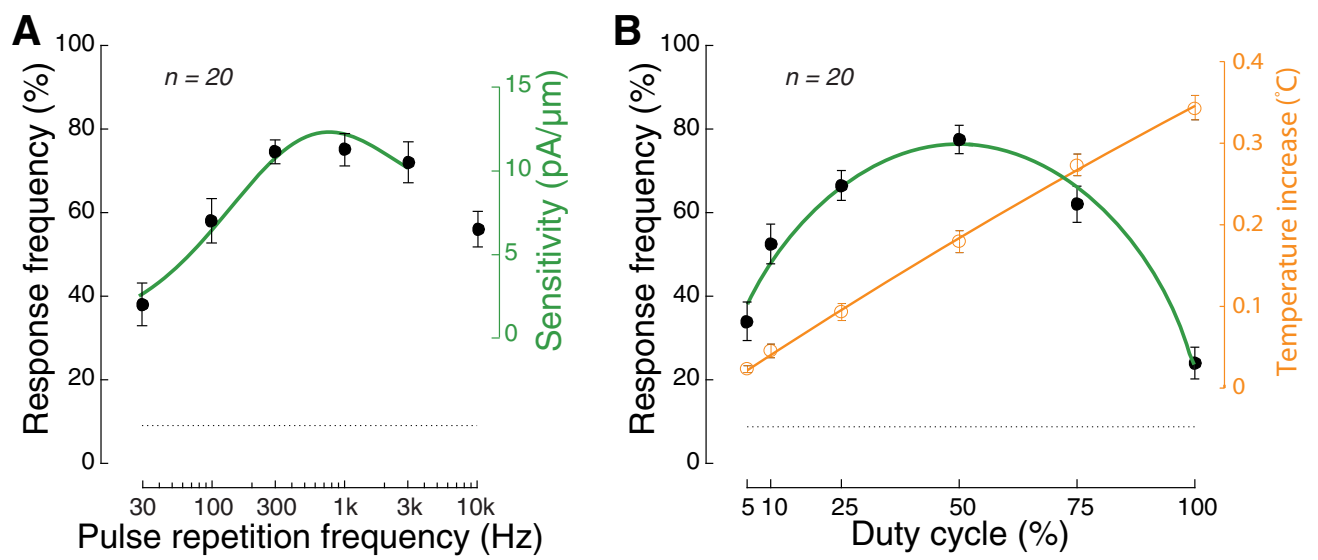

Figure 5. Ultrasound efficacy depends on pulse repetition frequency and duty cycle. $A$, The response (black circles, mean \pm SEM) of wild-type animals to a train of ultrasound pulses plotted as a function of pulse repetition frequency. The duty cycle was held constant at $50 \%$ in all cases, ensuring that all stimuli deliver the same amount of energy. The smooth curve (green) shows a simulation of the sensitivity of TRN currents to sinusoidal mechanical indentations (Eastwood et al., 2015). $\boldsymbol{B}$, The response (black circles, mean \pm SEM) of wild-type animals as a function of duty cycle. Because the pulse repetition rate was $1 \mathrm{kHz}$ in all cases, a duty cycle of 5, 10, 25, 50,75, 100\% corresponds to a pulse width of $50 \mu \mathrm{s}, 100 \mu \mathrm{s}, 250 \mu \mathrm{s}, 500 \mu \mathrm{s}, 750 \mu \mathrm{s}$, and $1 \mathrm{~ms}$ (continuous wave, no off epochs), respectively. The green curve shows the level of TRN activation expected from the frequency filtering shown in panel A (Materials and Methods for modeling details). Temperature increase (orange circles, mean $\pm \mathrm{SEM}, n=5$ ) as a function of duty cycle. The smooth curve (orange) is a quadratic fit to the data and is included for visual clarity. $\boldsymbol{A}, \boldsymbol{B}$, The carrier frequency was $10 \mathrm{MHz}$, the stimulus amplitude was $1 \mathrm{MPa}$, and the stimulus duration $200 \mathrm{~ms}$. The dotted line shows the unstimulated reversal frequency.

liver multiple discrete mechanical events into the tissue. To test this idea, we varied the duty cycle while holding stimulus duration, PRF, and pressure values constant at $200 \mathrm{~ms}, 1 \mathrm{kHz}$, and 1 $\mathrm{MPa}$, respectively. Figure $5 B$ shows the relationship between response frequency and duty cycle. It reveals that a duty cycle of $50 \%$ was more than threefold more potent than a $100 \%$ duty cycle $\left(77.5 \%\right.$ compared with $24.0 \%, p<10^{-12}, t$ test), even though a continuous stimulation delivers twice as much energy into the tissue as the pulsed protocol of $50 \%$ duty. In line with this finding, pulsed ultrasound stimulation has been found more effective than continuous stimulation in eliciting motor responses in rats (Kim et al., 2014). That study also found the value of $50 \%$ duty to be optimal. It is important to note that the $24.0 \%$ response rate for the continuous stimulus (100\% duty) is above baseline $(p<0.001, t$ test, $n=20)$. Thus, although a pulsed stimulus is more effective than a continuous stimulus, pulsing the ultrasound is not necessary to elicit a detectable response. The figure further shows that the width of the individual mechanical events associated with the ultrasound can be quite brief, just $50 \mu \mathrm{s}$ ( $5 \%$ of duty), and still trigger appreciable behavioral responses (response rate of $34.0 \%$, significantly different from baseline at $p<0.0001, t$ test, $n=20$ ). This is even though the energy delivered into the tissue is only $1 / 10$ th of that delivered at $50 \%$ duty.

Whereas heating increases linearly with duty cycle (Fig. $5 B$, orange plot), behavioral response frequency had a nonlinear dependence on duty cycle. The heating effect is expected from the fact that the energy delivered in the tissue increases with duty cycle. To ask whether or not the dependence on duty cycle could be explained by the frequency dependence of TRN activation, we simulated the frequency distribution expected as a function of duty cycle and combined with this the model from Eastwood et al. (2015). This model matched the experimental results (Fig. 5B, green line). Collectively, the effect of duty cycle reinforces the idea that behavioral responses to ultrasound are mediated by mechanical effects and not by heating.

\section{Discussion}

We sought to illuminate the biophysical mechanisms that underlie ultrasound stimulation of excitable cells. To do so, we used C. elegans as a model, harnessing its well-characterized and com- pact nervous system and comprehensive library of animals with specific genetic interventions. This animal has an extraordinary ability to detect tiny thermal fluctuations and mechanical stimuli (O'Hagan et al., 2005; Ramot et al., 2008). We found that ultrasound elicits robust reversal behaviors and that the response probability depends on stimulus intensity, duration, and specific pulsing protocols. Sensitivity to ultrasound and its modulation by pressure are preserved in mutants deficient in thermosensation and eliminated in mutants defective in mechanosensation. These findings are in agreement with a report (Zhou et al., 2017), showing that $\operatorname{tax}-4$ mutants, which are defective in thermosensing, retain the ability to respond to brief pulses of highfrequency ultrasound. Consistent with nonthermal, mechanical activation of sensory neurons linked to reversal behaviors, the response probability exhibited optima in both PRF and duty cycle.

Ultrasound-evoked reversal responses required expression of MEC-4, a key subunit of a touch-activated mechanosensitive ion channel. This finding implies that ultrasound can activate neurons by acting on mechanosensitive ion channels. Notably, because the MEC-4 ion channel complex is insensitive to changes in membrane voltage or capacitance, our findings are inconsistent with the hypothesis that ultrasound acts by inducing changes in membrane capacitance (Krasovitski et al., 2011; Plaksin et al., 2014).

We tested whether another mechanosensitive channel might contribute to ultrasound-evoked behaviors by analyzing strains carrying deletions in the trp-4 gene. Prior work showed that TRP-4 is a pore-forming subunit of mechanosensitive channel expressed by texture-sensing neurons in the worm's head (Kang et al., 2010) and suggested that this protein could sensitize neurons to ultrasound stimulation. Yet, we did not detect any effect of the loss of TRP-4 channels on ultrasound-evoked responses in three independent strains carrying validated deletions in the $\operatorname{trp}-4$ gene. We did detect a decrease in ultrasound sensitivity in a fourth strain (VC1141) that was used in a previous study (Ibsen et al., 2015). However, this phenotype is not due to loss of trp-4 function because four rounds of outcrossing eliminated it. Rather, the partial loss of ultrasound sensitivity is likely to be due to an unidentified mutation(s) in the VC1141 strain. Additional investi- 
gations will be needed to identify the affected gene(s), an effort that could reveal additional genetic factors regulating sensitivity to ultrasound stimulation.

This study provides evidence that ultrasound can stimulate neurons through its mechanical mode of action. Within the mechanical domain, there can be several specific candidate mechanisms at play. First, ultrasound may elicit cavitation, a phenomenon characterized by formation and collapse of gaseous bodies in liquid media or soft tissues. However, for frequencies $>1 \mathrm{MHz}$, cavitation requires pressures $>5 \mathrm{MPa}$ and the cavitation threshold for $10 \mathrm{MHz}$ is even higher (Nightingale et al., 2015). Thus, both the $10 \mathrm{MHz}$ transducer and low pressures we used make cavitation unlikely. Second, the incident tissue, such as a cell membrane, experiences oscillations with period equal to the ultrasound carrier frequency. The pressures used for neuromodulation can cause appreciable particle displacement (on the order of 0.01-0.1 $\mu \mathrm{m}$ ) (Gavrilov et al., 1976). Nonetheless, the displacement is distributed in sinusoidal fashion along the wavelength $(\sim 100 \mu \mathrm{m}$ at $10 \mathrm{MHz})$ of the propagating wave. This creates a very small displacement gradient (e.g., $0.1 \mu \mathrm{m}$ per 100 $\mu \mathrm{m})$. It is questionable whether such a small gradient can cause significant enough deformation of a pore segment of an ion channel with regard to the channel dimensions. Moreover, the primary pressure oscillations, which occur at a specific carrier frequency, cannot explain the frequency dependence of the responses (Fig. 5A). The third and most probable form of mechanical energy underlying the effects in this study is the acoustic radiation force (Trahey et al., 2004; Sarvazyan et al., 2010; Iversen et al., 2017). Acoustic radiation forces result from differences in acoustic intensities at individual points in space. The differences can be caused by ultrasound absorption, scattering, reflection, or other phenomena, and lead to net forces on the tissue (Duck et al., 1998). Acoustic radiation force exerts a steady pressure on a target throughout the time of ultrasound application. This steady pressure may stretch a cell membrane to an extent that affects conformation states of ion channels or other active molecules tied to the membrane. A simulation of the propagating ultrasound field, for the pressure of $1 \mathrm{MPa}$, revealed that a net acoustic radiation force of $873 \mathrm{nN}$ can act on the animal's head (see Materials and Methods). This exceeds the animal's sensitivity threshold to mechanical forces, which is $50-100 \mathrm{nN}$ (Petzold et al., 2013). Thus, the acoustic radiation force expected for a $1 \mathrm{MPa}$ stimulus is sufficient to engage the animal's mechanosensation. It is worth noting that the radiation force acts during the On epochs of the ultrasound (Fig. 1C, black rectangles), and not during the Off epochs when the ultrasound amplitude is zero. This way, pulsed ultrasound delivers force pulses at a specific PRF, and there can therefore be a modulation by the PRF (Fig. 5A).

Our findings implicate mechanical force as a major physical effect of ultrasound on neurons and their ion channels, delineating a complete pathway from mechanical force to activation of excitable cells. In this light, it is tempting to reiterate a potential unifying mechanism linking ultrasound to activation of excitable cells (Tyler, 2011). Suppose that ultrasound exerts similar mechanical effects in complex nervous tissues as it does in C. elegans. In addition, suppose that ultrasound deforms tissue and generates mechanical strain in neurons sufficient to activate mechanosensitive ion channels, as in C. elegans. Ion channels likely to subserve this function in mammals include the intrinsically mechanosensitive K2P family of potassium channels (Brohawn et al., 2014) and piezo channels (Syeda et al., 2016) known to be expressed in the brain. In support of this idea, the activity of $\mathrm{K} 2 \mathrm{P}$ channels, including TREK-1, TREK- 2, and TRAAK, is po- tentiated by ultrasound stimulation in heterologous cells (Kubanek et al., 2016). Sensitivity to the mechanical effects of ultrasound might not be limited to primarily mechanosensitive channels. For instance, voltage-gated sodium channels have been implicated in activation of neurons by ultrasound (Tyler, 2011, 2012; Kubanek et al., 2016) and are known to be sensitive to membrane tension (Beyder et al., 2010).

Exactly how ultrasound stimulation is translated into local mechanical strain will depend on the material properties of the excitable tissues under study. In the case of C. elegans, we found that the PRF and duty cycle dependence of ultrasound-evoked behaviors agreed with a model that links tissue indentation associated with a mechanical stimulus to the activation of MEC-4dependent channels (Eastwood et al., 2015). The correspondence (Fig. 5A) suggests that ultrasound-induced radiation force elicits a profile of mechanical strain similar to that produced by indentation with a physical probe. The model (Eastwood et al., 2015) also provides insight into why stimuli of $50 \%$ duty are the most potent (Fig. 5B): stimuli delivered at lower or higher duty cycle values contain a majority of their energy in high-frequency harmonics (relative to the $1 \mathrm{kHz} \mathrm{PRF}$ ), and these high-frequency harmonics are filtered out by the tissue (Fig. 5A). However, the relationship between bursts of ultrasound pulses and the mechanical strain we infer that it generates is not currently known, and future measurements of ultrasound-induced strain will be needed to fill this knowledge gap for worms and other tissues. Consistent with the proposal that tissues differ in their mechanical filtering properties, ultrasound-evoked behaviors have an optimal PRF near $500 \mathrm{~Hz}$ in C. elegans and show strong effects at $~ 3000$ $\mathrm{Hz}$ in mice (King et al., 2013). These results indicate that an improved understanding of mechanical filtering by soft tissues will be needed to further the long-term goal of applying ultrasound as a noninvasive modality to stimulate excitable cells.

This study reveals that pulsatile forces associated with ultrasound are potent enough to activate mechanically sensitive ion channels in living animals. Given that many ion channels expressed in neurons and glia are mechanically sensitive (Ostrow and Sachs, 2005; Tyler, 2012), this study illuminates one way that ultrasound could influence activity in the brain. Another way would be to sensitize specific neurons to ultrasound through ectopic expression of a channel known to be mechanosensitive, a strategy referred to as sonogenetics (Ibsen et al., 2015). In both scenarios, our work underscores the importance of tuning stimulus parameters to maximize acoustic radiation force and may help to further the development of ultrasound as a noninvasive and spatially precise tool to study the nervous system and potentially to ameliorate neurological disorders.

\section{References}

Altman PL, Dittmer DS, Zwemer RL (1974) Biology data book. Bethesda, MD: Federation of American Societies for Experimental Biology.

Barber TW, Brockway JA, Higgins LS (1970) The density of tissues in and about the head. Acta Neurol Scand 46:85-92. CrossRef Medline

Beyder A, Rae JL, Bernard C, Strege PR, Sachs F, Farrugia G (2010) Mechanosensitivity of nav1. 5, a voltage-sensitive sodium channel. J Physiol 588:4969-4985. CrossRef Medline

Brohawn SG, Su Z, MacKinnon R (2014) Mechanosensitivity is mediated directly by the lipid membrane in TRAAK and TREK $1 \mathrm{~K}^{+}$channels. Proc Natl Acad Sci U S A 111:3614-3619. CrossRef Medline

Buiochi EB, Miller RJ, Hartman E, Buiochi F, Bassani RA, Costa ET, O’Brien WD (2012) Transthoracic cardiac ultrasonic stimulation induces a negative chronotropic effect. IEEE Trans Ultrason Ferroelectr Freq Control 59:2655-2661. CrossRef Medline

Colucci V, Strichartz G, Jolesz F, Vykhodtseva N, Hynynen K (2009) Fo- 
cused ultrasound effects on nerve action potential in vitro. Ultrasound Med Biol 35:1737-1747. CrossRef Medline

Dalecki D (2004) Mechanical bioeffects of ultrasound. Annu Rev Biomed Eng 6:229-248. CrossRef Medline

Deffieux T, Younan Y, Wattiez N, Tanter M, Pouget P, Aubry JF (2013) Low-intensity focused ultrasound modulates monkey visuomotor behavior. Curr Biol 23:2430-2433. CrossRef Medline

Diaz-Franulic I, Poblete H, Min-o-Galaz G, González C, Latorre R (2016) Allosterism and structure in thermally activated transient receptor potential channels. Annu Rev Biophysics 45:371-398. CrossRef Medline

Driscoll M, Chalfie M (1991) The mec-4 gene is a member of a family of Caenorhabditis elegans genes that can mutate to induce neuronal degeneration. Nature 349:588-593. CrossRef Medline

Duck FA, Baker AC, Starritt HC (1998) Ultrasound in medicine. Boca Raton, FL: CRC.

Eastwood AL, Sanzeni A, Petzold BC, Park SJ, Vergassola M, Pruitt BL, Goodman MB (2015) Tissue mechanics govern the rapidly adapting and symmetrical response to touch. Proc Natl Acad Sci U S A 112:E6955-E6963. CrossRef Medline

Foster KR, Wiederhold ML (1978) Auditory responses in cats produced by pulsed ultrasound. J Acoust Soc Am 63:1199-1205. CrossRef Medline

Fry F, Ades HW, Fry WJ (1958) Production of reversible changes in the central nervous system by ultrasound. Science 127:83-84. CrossRef Medline

Garrity PA, Goodman MB, Samuel AD, Sengupta P (2010) Running hot and cold: behavioral strategies, neural circuits, and the molecular machinery for thermotaxis in C. elegans and Drosophila. Genes Dev 24:2365-2382. CrossRef Medline

Gavrilov LR, Gersuni GV, Ilyinsky OB, Sirotyuk MG, Tsirulnikov EM, Shchekanov EE (1976) The effect of focused ultrasound on the skin and deep nerve structures of man and animal. Prog Brain Res 43:279-292. CrossRef Medline

Gavrilov LR, Tsirulnikov EM, Davies IA (1996) Application of focused ultrasound for the stimulation of neural structures. Ultrasound Med Biol 22:179-192. CrossRef Medline

Glauser DA, Goodman MB (2016) Molecules empowering animals to sense and respond to temperature in changing environments. Curr Opin Neurobiol 41:92-98. CrossRef Medline

Harvey EN (1929) The effect of high frequency sound waves on heart muscle and other irritable tissues. Am J Physiol 91:284-290. CrossRef

Husson SJ, Costa WS, Schmitt C, Gottschalk A (2005) Keeping track of worm trackers. WormBook 22:1-17. CrossRef Medline

Ibsen S, Tong A, Schutt C, Esener S, Chalasani SH (2015) Sonogenetics is a non-invasive approach to activating neurons in Caenorhabditis elegans. Nat Commun 6:8264. CrossRef Medline

Iversen MM, Christensen DA, Parker DL, Holman HA, Chen J, Frerck MJ, Rabbitt RD (2017) Low-intensity ultrasound activates vestibular otolith organs through acoustic radiation force. J Acoust Soc Am 141:42094219. CrossRef Medline

Kang L, Gao J, Schafer WR, Xie Z, Xu XZ (2010) C. elegans TRP family protein TRP-4 is a pore-forming subunit of a native mechanotransduction channel. Neuron 67:381-391. CrossRef Medline

Kim H, Chiu A, Lee SD, Fischer K, Yoo SS (2014) Focused ultrasoundmediated non-invasive brain stimulation: examination of sonication parameters. Brain Stimul 7:748-756. CrossRef Medline

King RL, Brown JR, Newsome WT, Pauly KB (2013) Effective parameters for ultrasound-induced in vivo neurostimulation. Ultrasound Med Biol 39:312-331. CrossRef Medline

Krasovitski B, Frenkel V, Shoham S, Kimmel E (2011) Intramembrane cavitation as a unifying mechanism for ultrasound-induced bioeffects. Proc Natl Acad Sci U S A 108:3258-3263. CrossRef Medline

Kremkau FW, Barnes RW, McGraw CP (1981) Ultrasonic attenuation and propagation speed in normal human brain. J Acoust Soc Am 70:29-38. CrossRef

Kubanek J, Shi J, Marsh J, Chen D, Deng C, Cui J (2016) Ultrasound modulates ion channel currents. Sci Rep 6:24170. CrossRef Medline

Lee W, Kim HC, Jung Y, Chung YA, Song IU, Lee JH, Yoo SS (2016) Transcranial focused ultrasound stimulation of human primary visual cortex. Sci Rep 6:34026. CrossRef Medline

Li W, Feng Z, Sternberg PW, Xu XZ (2006) A C. elegans stretch receptor neuron revealed by a mechanosensitive TRP channel homologue. Nature 440:684-687. CrossRef Medline
Menz MD, Oralkan O, Khuri-Yakub PT, Baccus SA (2013) Precise neural stimulation in the retina using focused ultrasound. J Neurosci 33:4550 4560. CrossRef Medline

Meyers R, Fry WJ, Fry FJ, Dreyer LL, Schultz DF, Noyes RF (1959) Early experiences with ultrasonic irradiation of the pallidofugal and nigral complexes in hyperkinetic and hypertonic disorders. J Neurosurg 16:32-54. CrossRef Medline

Mihran RT, Barnes FS, Wachtel H (1990) Temporally-specific modification of myelinated axon excitability in vitro following a single ultrasound pulse. Ultrasound Med Biol 16:297-309. CrossRef Medline

Morris P, Hurrell A, Shaw A, Zhang E, Beard P (2009) A Fabry-Perot fiberoptic ultrasonic hydrophone for the simultaneous measurement of temperature and acoustic pressure. J Acoust Soc Am 125:3611-3622. CrossRef Medline

Naor O, Krupa S, Shoham S (2016) Ultrasonic neuromodulation. J Neural Eng 13:031003. CrossRef Medline

Nightingale KR, Church CC, Harris G, Wear KA, Bailey MR, Carson PL, Jiang H, Sandstrom KL, Szabo TL, Ziskin MC (2015) Conditionally increased acoustic pressures in nonfetal diagnostic ultrasound examinations without contrast agents: a preliminary assessment. J Ultrasound Med 34:1-41. CrossRef Medline

O'Hagan R, Chalfie M, Goodman MB (2005) The MEC-4 DEG/ENaC channel of Caenorhabditis elegans touch receptor neurons transduces mechanical signals. Nat Neurosci 8:43-50. CrossRef Medline

Ostrow LW, Sachs F (2005) Mechanosensation and endothelin in astrocytes: hypothetical roles in CNS pathophysiology. Brain Res Rev 48:488508. CrossRef Medline

Petzold BC, Park SJ, Mazzochette EA, Goodman MB, Pruitt BL (2013) MEMS-based force-clamp analysis of the role of body stiffness in C. elegans touch sensation. Integr Biol (Camb) 5:853-864. CrossRef Medline

Plaksin M, Shoham S, Kimmel E (2014) Intramembrane cavitation as a predictive bio-piezoelectric mechanism for ultrasonic brain stimulation. Phys Rev 4:011004.

Rabbitt RD, Brichta AM, Tabatabaee H, Boutros PJ, Ahn J, Della Santina CC, Poppi LA, Lim R (2016) Heat pulse excitability of vestibular hair cells and afferent neurons. J Neurophysiol 116:825-843. CrossRef Medline

Ramot D, Johnson BE, Berry TL Jr, Carnell L, Goodman MB (2008) The parallel worm tracker: a platform for measuring average speed and druginduced paralysis in nematodes. PLoS One 3:e2208. CrossRef Medline

Ramot D, MacInnis BL, Goodman MB (2008) Bidirectional temperaturesensing by a single thermosensory neuron in C. elegans. Nat Neurosci 11:908-915. CrossRef Medline

Sarvazyan AP, Rudenko OV, Nyborg WL (2010) Biomedical applications of radiation force of ultrasound: historical roots and physical basis. Ultrasound Med Biol 36:1379-1394. CrossRef Medline

Sassaroli E, Vykhodtseva N (2016) Acoustic neuromodulation from a basic science prospective. J Ther Ultrasound 4:17. CrossRef Medline

Schafer WR (2015) Mechanosensory molecules and circuits in C. elegans. Pflugers Arch 467:39-48. CrossRef Medline

Schneider ER, Anderson EO, Gracheva EO, Bagriantsev SN (2014) Temperature sensitivity of two-pore (K2P) potassium channels. Curr Top Membr 74:113-133. CrossRef Medline

Stiernagle T (2006) Maintenance of C. elegans. WormBook 11:1-11. CrossRef Medline

Sukharev S, Corey DP (2004) Mechanosensitive channels: multiplicity of families and gating paradigms. Sci STKE 2004:re4. CrossRef Medline

Syeda R, Florendo MN, Cox CD, Kefauver JM, Santos JS, Martinac B, Patapoutian A (2016) Piezo1 channels are inherently mechanosensitive. Cell Rep 17:1739-1746. CrossRef Medline

Trahey GE, Palmeri ML, Bentley RC, Nightingale KR (2004) Acoustic radiation force impulse imaging of the mechanical properties of arteries: in vivo and ex vivo results. Ultrasound Med Biol 30:1163-1171. CrossRef Medline

Treeby BE, Cox BT (2010) k-Wave: MATLAB toolbox for the simulation and reconstruction of photoacoustic wave fields. J Biomed Opt 15: 021314. CrossRef Medline

Tsui PH, Wang SH, Huang CC (2005) In vitro effects of ultrasound with different energies on the conduction properties of neural tissue. Ultrasonics 43:560-565. CrossRef Medline

Tufail Y, Matyushov A, Baldwin N, Tauchmann ML, Georges J, Yoshihiro A, Tillery SI, Tyler WJ (2010) Transcranial pulsed ultrasound stimulates intact brain circuits. Neuron 66:681-694. CrossRef Medline 
Tufail Y, Yoshihiro A, Pati S, Li MM, Tyler WJ (2011) Ultrasonic neuromodulation by brain stimulation with transcranial ultrasound. Nat Protoc 6:1453-1470. CrossRef Medline

Tyler WJ (2011) Noninvasive neuromodulation with ultrasound? A continuum mechanics hypothesis. Neuroscientist 17:25-36. CrossRef Medline

Tyler WJ (2012) The mechanobiology of brain function. Nat Rev Neurosci 13:867-878. CrossRef Medline

Wasserman SM, Beverly M, Bell HW, Sengupta P (2011) Regulation of response properties and operating range of the AFD thermosensory neurons by cGMP signaling. Curr Biol 21:353-362. CrossRef Medline

Way JC, Chalfie M (1989) The mec-3 gene of Caenorhabditis elegans requires its own product for maintained expression and is expressed in three neuronal cell types. Genes Dev 3:1823-1833. CrossRef Medline

Yan Z, Zhang W, He Y, Gorczyca D, Xiang Y, Cheng LE, Meltzer S, Jan LY,
Jan YN (2013) Drosophila NOMPC is a mechanotransduction channel subunit for gentle-touch sensation. Nature 493:221-225. CrossRef Medline

Ye PP, Brown JR, Pauly KB (2016) Frequency dependence of ultrasound neurostimulation in the mouse brain. Ultrasound Med Biol 42:15121530. CrossRef Medline

Yoo SS, Bystritsky A, Lee JH, Zhang Y, Fischer K, Min BK, McDannold NJ, Pascual-Leone A, Jolesz FA (2011) Focused ultrasound modulates region-specific brain activity. Neuroimage 56:1267-1275. CrossRef Medline

Zhou W, Wang J, Wang K, Huang B, Niu L, Li F, Cai F, Chen Y, Liu X, Zhang X, Cheng H, Kang L, Meng L, Zheng H (2017) Ultrasound neuromodulation chip: activation of sensory neurons in Caenorhabditis elegans by surface acoustic waves. Lab Chip 17:1725-1731. CrossRef Medline 